
\title{
3 Research Square \\ A Case of Pasteurella Multocida Pneumonia, Bacteremia, and Septic Shock
}

Wesley Tang ( $\nabla$ wctang24@gmail.com )

Kettering Medical Center https://orcid.org/0000-0002-8878-3673

\section{Sulagna Das}

Kettering Medical Center

James Galbraith

South Dayton Acute Care Consultants

\section{Research Article}

Keywords: Pasteurella, pneumonia, bacteremia

Posted Date: September 7th, 2021

DOI: https://doi.org/10.21203/rs.3.rs-315527/v1

License: (1) This work is licensed under a Creative Commons Attribution 4.0 International License. Read Full License

Version of Record: A version of this preprint was published at SN Comprehensive Clinical Medicine on January 10th, 2022. See the published version at https://doi.org/10.1007/s42399-021-01090-0. 


\section{Abstract}

Pasteurella multocida is a ubiquitous organism found in the oropharynx of healthy domestic animals, especially dogs and cats. It is most known as a human pathogen, reported after animal bite incidents. In atraumatic infections, Pasteurella has been associated with patients in an immunocompromised state, such as those with Hodgkin's lymphoma, patients with known cirrhosis, or those with preexisting cavitary pulmonary lesions. It is rare to isolate Pasteurella in an immunocompetent patient without known trauma. Here, we present a case of Pasteurella multocida pneumonia, bacteremia, and septic shock in an individual without a history of an animal bite, with a review of relevant literature.

\section{Introduction}

Pasteurella multocida is a Gram-negative, facultative anaerobe, with coccobacilli morphology that is commonly found in the oropharynx of cats and dogs[1]. The carriage rate has been reported to be approximately $80 \%$ in cats, and $40 \%$ in dogs[2]. The organism was first described by Louis Pasteur in $1880[3]$. Typically, the organism is introduced into the human body via cat/dog bites. Most common infections have been associated with skin and soft tissue infections such as cellulitis, with more serious infections escalating to septic arthritis, and osteomyelitis[4]. More severe infections have been associated with individuals with cirrhosis, hematological malignancies, or patients with history of organ transplantation $[5,6]$. In patients with Pasteurella bacteremia, mortality rates have been documented as high as $30 \%[7]$.

We describe a case of an immunocompetent patient, without known history of a dog or cat bite, with resultant Pasteurella pneumonia, with subsequent bacteremia, and septic shock. The purpose of this article is to raise awareness that Pasteurella may present more invasively than previously documented.

\section{Case Presentation}

A 77-year-old male with multiple comorbidities including a history of left adrenal mass, disseminated histoplasmosis status post left adrenalectomy and left nephrectomy, chronic pleural effusions, renal failure on hemodialysis, chronic obstructive pulmonary disease, history of perforated diverticulitis-status post colectomy in the recent past, presented to the emergency department with pain in the left side of his abdomen for the last 18 hours. Patient stated that he worked in his garage throughout the day and as the day concluded, he noted some vague left-sided discomfort. He stated the pain seemed to be positional and seemed to be worsened by deep breathing or coughing. He denies any known injury that occurred during the day. Patient has had some chronic shortness of breath and cough due to ongoing histoplasmosis and a chronic left pleural effusion. Patient stated that approximately 03:00 that morning he was awakened with some worsening pain. Again he stated the pain seemed to be positional and worsened by deep breathing or coughing. Patient stated he felt somewhat bloated and had a bowel movement at that time which he described as a normal bowel movement. Patient stated that his discomfort had persisted and it has led to his presentation. A CT scan of his abdomen/pelvis was 
subsequently performed given his history of perforated diverticulitis. This was read as non-acute, though the lung bases did show possible pneumonia. He was then discharged with instructions to follow up with his primary care provider. When he was taken back by his family, he was reported to be unresponsive in the car and was quickly brought back by the family to a different emergency department. Further history elicited from the family revealed that the patient lived with 2 cats, without any reported bites, scratches, or wounds.

On admission, patient was noted to be unresponsive and subsequently was intubated. His arterial blood gas would show pH 7.10, PCO2 69, PO2 65, saturations at $82 \%$ on $100 \%$ FiO2. Laboratory workup was significant for leukocytosis of $30.7 \mathrm{k} / \mathrm{uL}$, BUN $51 \mathrm{mg} / \mathrm{dL}$, creatinine $2.8 \mathrm{mg} / \mathrm{dL}$ (baseline 1.4), AST 522 $\mathrm{U} / \mathrm{L}$, ALT $627 \mathrm{U} / \mathrm{L}$, alkaline phosphatase $77 \mathrm{U} / \mathrm{L}$, and total bilirubin $1.4 \mathrm{mg} / \mathrm{dL}$. Blood cultures from the first hospital visit would later result positive for Pasteurella multocida. Likewise, a bronchoalveolar lavage performed during hospitalization also later resulted positive for Pasteurella multocida. The patient was hypotensive with blood pressures measuring 70 s/40s and he was started on norepinephrine and vancomycin piperacillin/tazobactam. Fluid resuscitation was also performed with $2.2 \mathrm{~L}$ of crystalloid fluid provided. Later the patient developed evidence of shock liver, acute kidney injury, profound lymphocytosis with neutropenia, and severe lactic acidosis. Nephrology, hematology, surgery, infectious disease services were consulted. As the patient's condition was deteriorating, piperacillin/tazobactam and vancomycin were discontinued and meropenem instead started. The patient showed slow improvement during his stay in the ICU, and was gradually able to be weaned from vasopressors and mechanical ventilation. Antibiotics were switched to ampicillin/sulbactam, and the patient would be transferred to a medical/surgical unit from ICU. He was found to have a lower extremity deep vein thrombosis during this time, and an IVC filter was placed, given marked thrombocytopenia. Unexpectedly and after a few days in the medical/surgical unit, the patient once again developed respiratory failure and severe hypotension. The patient was reintubated and taken back to the ICU. Despite aggressive measures including continuous renal replacement therapy and vasopressors, the patient once more reentered a shock state with multiorgan dysfunction. Escalating pressor requirements, CRRT, mechanical ventilatory support were all maximally applied. A family meeting was held and the family decided to pursue comfort care for the patient. The patient expired two days after the decision to pursue comfort care through pulseless electrical activity and asystole.

\section{Conclusions}

P. multocida is common among domestic animals. Dog or cat ownership in the United States is estimated to be approximately $30 \%-40 \%$. In general, most human infections are a result of animal bites or scratches primarily involving the skin and soft tissue. An estimated $1 \%$ of all emergency department visits per year are associated with animal bites[8]. Although there are a few cases of $P$ multocida implicated in more serious infections such as bacteremia, peritonitis, and meningitis, these are exceedingly rare[2, 4-6, 9]. Factors associated with systemic involvement include cavitary lung pathology, immunocompromised states, patients on dialysis, and patients with malignancy[8]. 
In this case report, we present an immunocompetent patient with $P$. multocida infection, with no documented history of inciting bite/scratch, with subsequent pneumonia, bacteremia, and septic shock. We postulate that our patient was likely exposed to secretions from his two cats through the inhalation of his pet's aerosols. This would later develop into pneumonia.

Because of the high mortality rate associated with Pasteurella bacteremia, clinicians should be alert to the possibility of severe infection in patients with exposure to cats and dogs, regardless of history of bite or scratch wounds. Likewise, clinicians should obtain a detailed travel and animal exposure history toward the diagnosis of Pasteurella pneumonia. In counseling patients, clinicians should advise their patients who may be immunocompromised, frail, or have underlying chronic pulmonary disease to avoid close animal contact.

\section{Declarations}

\section{Funding Support}

None

\section{Conflicts of Interest}

The authors declare that they do not have a conflict of interest

\section{Ethics Approval \& Consent to Participate}

Kettering Health Network Institutional Review Board oversees all scholarly activities across its facilities and determined that case reports meet the criteria for exempt review, and do not meet the definition of human research. Approval and consent provided.

\section{Consent for Publication}

Consent to publish was obtained from the patient's next of kin

\section{Availability of Data and Material}

Not applicable

Code Availability

Not applicable

\section{Author Contributions}

WT \& SD designed and conducted the research. JG provided the data. WT had primary responsibility for final content. All authors read and approved the final manuscript. 


\section{References}

1. Aljameely, A. and G. Wali, Pasteurella multocida Septic Shock: Case Report and Literature Review. Case Rep Infect Dis, 2019. 2019: p. 1964161.

2. van der Reijden, M., et al., Infection of lung cavitations in a young dog owner with Hodgkin's lymphoma caused by Pasteurella multocida, without a dog bite: confirmed zoonotic transmission by tagmentation microbiome analysis. BMJ Case Rep, 2018. 11(1).

3. Dresselaars, H.F., et al., Peritoneal dialysis-associated peritonitis of zoonotic origin, when minor gets major. Neth J Med, 2014. 72(10): p. 551-3.

4. Mirzai, S., et al., A Case Report on Pasteurella multocida Peritoneal Dialysis-Associated Peritonitis: When Cats Think Medical Equipment Are Toys. Case Rep Nephrol, 2019. 2019: p. 5150695.

5. Yokose, N. and K. Dan, Pasteurella multocida sepsis, due to a scratch from a pet cat, in a postchemotherapy neutropenic patient with non-Hodgkin lymphoma. Int J Hematol, 2007. 85(2): p. 146-8.

6. Migliore, E., et al., Pasteurella multocida infection in a cirrhotic patient: case report, microbiological aspects and a review of literature. Adv Med Sci, 2009. 54(1): p. 109-12.

7. Ebright, J., A.B. Frey, and M.R. Fairfax, Pasteurella multocida Infections and Bacteremia: A TwentyYear Experience at an Urban Medical Center. Infectious Diseases in Clinical Practice, 2009. 17(2): p. 102-104.

8. Giordano, A., et al., Clinical Features and Outcomes of Pasteurella multocida Infection. Medicine (Baltimore), 2015. 94(36): p. e1285.

9. Kofteridis, D.P., et al., Bacteremic community-acquired pneumonia due to Pasteurella multocida. Int J Infect Dis, 2009. 13(3): p. e81-3.

\section{Figures}




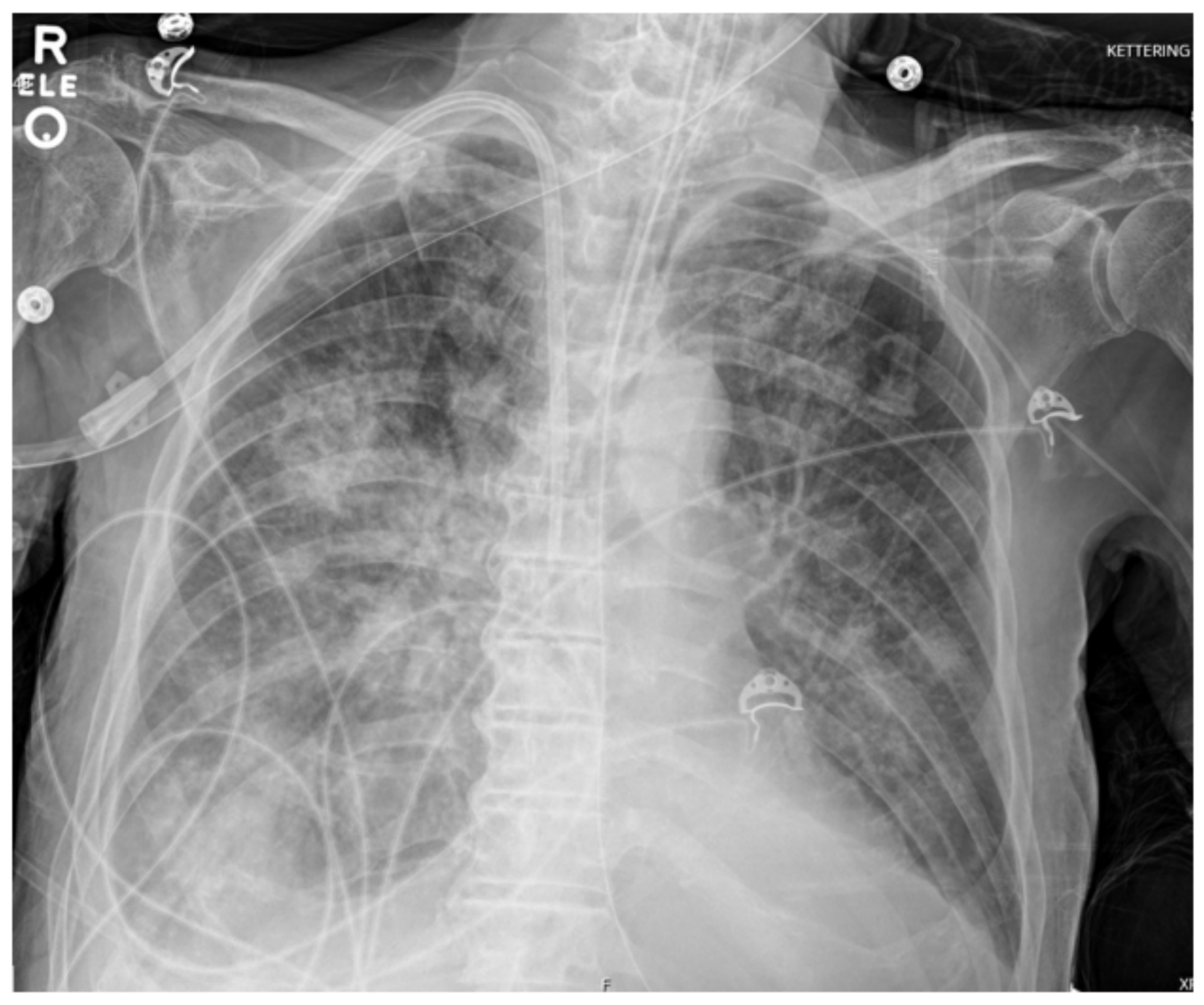

Figure 1

Chest Xray demonstrating bilateral widespread airspace disease 


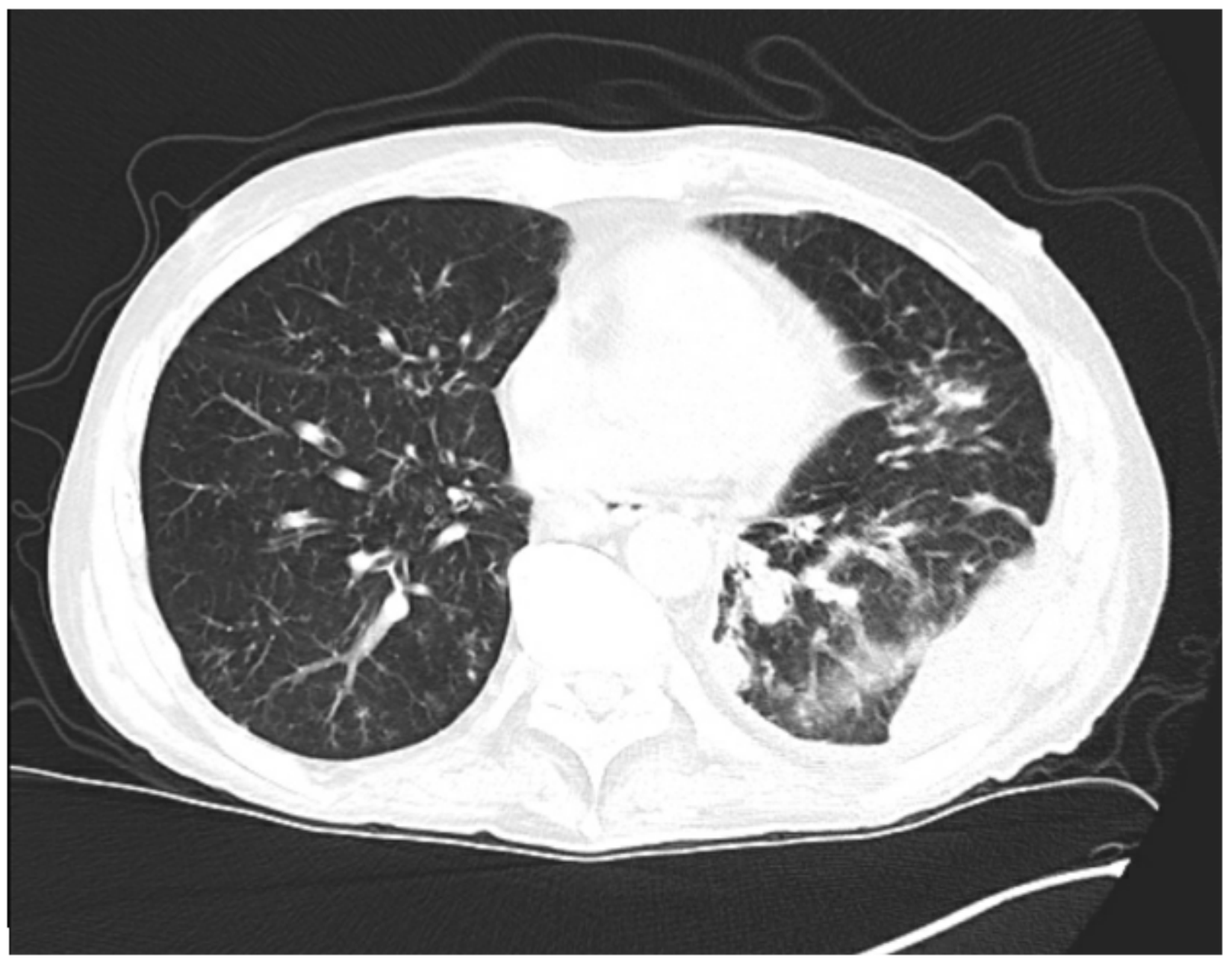

Figure 2

Computed tomography demonstrating multifocal airspace disease within the lungs bilaterally. Mucous plugging in the left lower lobe and lingula. 